\title{
Exact Solution for Long-Term Size Exclusion Suspension-Colloidal Transport in Porous Media
}

\author{
Z. You, ${ }^{1}$ P. Bedrikovetsky, ${ }^{1}$ and L. Kuzmina ${ }^{2}$ \\ ${ }^{1}$ Australian School of Petroleum, The University of Adelaide, Adelaide, SA 5005, Australia \\ ${ }^{2}$ National Research University Higher School of Economics, 20 Myasnitskaya Ulitsa, Moscow 101000, Russia \\ Correspondence should be addressed to Z. You; zhenjiang.you@adelaide.edu.au
}

Received 3 September 2013; Accepted 26 November 2013

Academic Editor: Shuyu Sun

Copyright (c) $2013 \mathrm{Z}$. You et al. This is an open access article distributed under the Creative Commons Attribution License, which permits unrestricted use, distribution, and reproduction in any medium, provided the original work is properly cited.

\begin{abstract}
Long-term deep bed filtration in porous media with size exclusion particle capture mechanism is studied. For monodispersed suspension and transport in porous media with distributed pore sizes, the microstochastic model allows for upscaling and the exact solution is derived for the obtained macroscale equation system. Results show that transient pore size distribution and nonlinear relation between the filtration coefficient and captured particle concentration during suspension filtration and retention are the main features of long-term deep bed filtration, which generalises the classical deep bed filtration model and its latter modifications. Furthermore, the exact solution demonstrates earlier breakthrough and lower breakthrough concentration for larger particles. Among all the pores with different sizes, the ones with intermediate sizes (between the minimum pore size and the particle size) vanish first. Total concentration of all the pores smaller than the particles turns to zero asymptotically when time tends to infinity, which corresponds to complete plugging of smaller pores.
\end{abstract}

\section{Introduction}

Transport, filtration, and subsequent retention of suspended particles and colloids in porous media are common phenomena in nature and in many industrial applications. In petroleum industry, migration of fine particles in low consolidated natural rocks during production of heavy oils leads to significant productivity decline [1-4]. Invasion of drilling fluid into oil and gas reservoirs results in well impairment and formation damage due to particle straining in thin pores [57]. Flow of suspensions and colloids in porous media with particle retention by matrix is also important for filtering of water and industrial liquid or gas fluid streams [8-10], enhanced bioremediation [11, 12], particle accumulation in the streambed sediments [13], propagation of biocolloids (bacteria viruses and protozoan parasites) in aquifers, surface water and wastewater treatment, and so forth [14-20].

A thorough understanding and reliable prediction of particle transport and retention by mathematical modelling are critical to the planning and design of abovementioned industrial and environmental processes. Up to date, considerable research has been devoted to the description of transport and retention behaviour of suspensions in porous media, as well as their effects on the alteration of the formation rock (a number of reviews are available in the literature; see [2129]). In spite of all these works, the mechanisms of suspension transport and capture in porous media are still incompletely understood and quantified.

The classical suspension-colloidal deep bed filtration (DBF) theory is the most commonly used approach for predicting particle transport behaviour and the consequent media alterations $[3,8,9,14,15]$. The classical DBF model includes two equations for particle population balance and capture kinetics, respectively $[21,30]$. Several forms of filtration coefficient as function of retained particle concentration for different capture mechanisms and the resultant analytical solutions have been reported in the literature [21, 31]. However, the reported mismatch between the modelled and measured particle DBF data makes it necessary to examine the fundamental principles of the classical model for suspension transport in porous media, including its upscaling from micro- to macroscale and possible model generalisations [32-34]. 


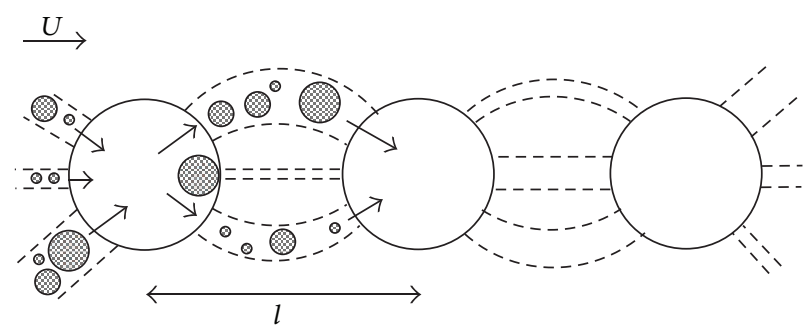

(a)

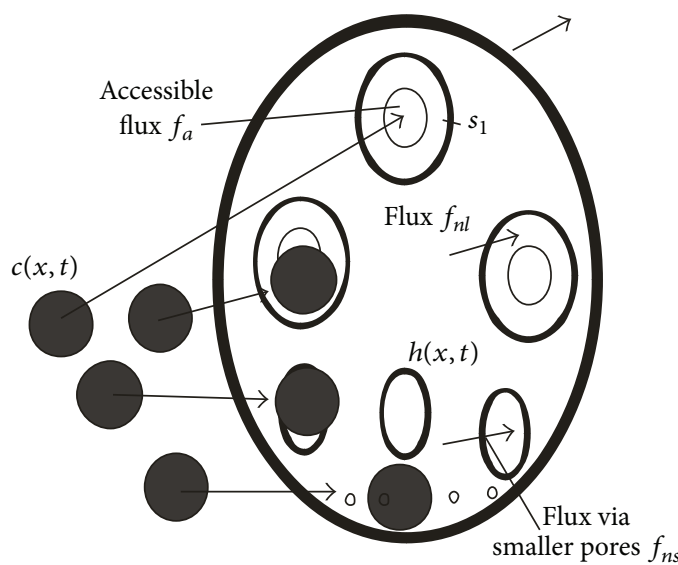

(b)

Figure 1: Geometric model of parallel capillary bundles with mixing chambers: (a) bundles of parallel capillaries alternated by mixing chambers; (b) cross section of the capillary bungle with size-excluded particles.

Sharma and Yortsos [35-37] derived a new population balance model, accounting for variation of pore and particle size distributions due to several particle capture mechanisms. It is assumed that the particle population moves at the average flow velocity of the carrier fluid, and the whole porous space is accessible to particles. The particles smaller than the pores pass without straining; while the particles larger than the pores are size-excluded in the medium. Particularly, these assumptions lead to independent deep bed filtration of polydispersed particles under the low retention conditions.

Introduction of accessibility and flux reduction factors into the population balance equations describes simultaneous flow of suspension in accessible pores and flow of particlefree water in inaccessible fraction of porous space; it also results in the particle speed that differs from the carrier water velocity [32, 38, 39]. A linearised model describes deep bed filtration under the short-time injection; the concentration decays with time exponentially due to retention $[14,15,21,23]$. The analytical model for long-time injection does not account for accessibility and flux reduction factors $[38,39]$.

In the present paper, an exact solution for long-term deep bed filtration accounting for accessibility and flux reduction factors is derived. Being downscaled, the solution exhibits the transient development of the pore size distribution due to particle size exclusion. The macroscale equations result in nonlinear retained-particle-concentration dependencies for filtration coefficient as well as the accessibility and flux reduction factors, which generalise the classical DBF model.

The structure of this paper is as follows. Section 2 presents the microscale system of governing equations with varying pore and particle size distributions. The upscaling of the micromodel for monodispersed suspension is briefly derived in Section 3. The analytical solution of the upscaled equations with expressions for suspended and captured particle concentrations during long-term injection is derived in Section 4. The concentration profiles and histories as obtained from the exact solution along with constitutive relations of the upscaled model are presented and discussed in Section 5. Finally, Section 6 presents the conclusions of the study.

\section{Microstochastic Model for Suspension Transport in Porous Media}

The geometric model of porous media for size exclusion suspension-colloidal transport is a bundle of parallel tubes intercalated by the mixing chambers (Figure 1(a)). Size exclusion flow in any arbitrary 3D domain occurs with particle motion in "thicker" pore and capture in "thinner" pore throat. The parallel-tube-mixing-chamber model (PTMC) separates flow from capture: the capture occurs at the exit of mixing chambers at entrances of thinner pores, while the motion occurs in larger parallel capillaries (Figure 1(b)). The phenomenological parameters and functions used in the stochastic model are described briefly in this section. For more details, refer to [32].

The number of pores per unit cross-sectional area of porous media is defined by the pore concentration function

$$
h(x, t)=\int_{0}^{\infty} H\left(r_{p}, x, t\right) d r_{p}
$$

which is the integral of pore concentration distribution function $H\left(r_{p}, x, t\right)$ in terms of the pore radius $r_{p}$. In the similar manner, the overall concentration of suspended particles in porous media results from the integration of concentration of these particles in $r_{s}$ for all particle sizes

$$
c(x, t)=\int_{0}^{\infty} C\left(r_{s}, x, t\right) d r_{s}
$$

where $C\left(r_{s}, x, t\right)$ is the concentration distribution function of suspended particles. The total concentration of retained particles is also obtained by integration in particle size

$$
\sigma(x, t)=\int_{0}^{\infty} \Sigma\left(r_{s}, x, t\right) d r_{s}
$$


where $\Sigma\left(r_{s}, x, t\right)$ is the concentration distribution function of retained particles.

The porosity is the total cross-sectional area of all pores per unit cross-sectional area of porous media

$$
\phi[H]=\int_{0}^{\infty} s_{1}\left(r_{p}\right) H\left(r_{p}, x, t\right) d r_{p},
$$

where $s_{1}\left(r_{p}\right)=\pi r_{p}^{2}$ is the cross-sectional area of a single pore with radius $r_{p}$. The accessible fraction of the total porosity can be obtained by integrating the cross-sectional area of large pores only $\left(r_{p}>r_{s}\right)$,

$$
\phi_{a}\left[H, r_{s}\right]=\int_{r_{s}}^{\infty} s_{1}\left(r_{p}\right) H\left(r_{p}, x, t\right) d r_{p},
$$

due to the fact that only the pores with sizes larger than $r_{s}$ are accessible to these particles.

The conductance in a single pore follows from the steady state Poiseuille flow in circular capillary [40], expressed as $k_{1}\left(r_{p}\right)=\pi r_{p}^{4} / 8$. Comparison with Darcy's law leads to the following formula for permeability:

$$
k[H]=\int_{0}^{\infty} k_{1}\left(r_{p}\right) H\left(r_{p}, x, t\right) d r_{p} .
$$

The fractions of accessible and inaccessible fluxes are defined as the accessible and inaccessible fractional flow functions, respectively. The total flux of flow is the sum of the flux via accessible larger pores and that via inaccessible smaller pores, for any given particle size $r_{s}$. According to Darcy's law, the accessible fractional flow function can be calculated as fraction of accessible permeability

$$
f_{a}\left[H, r_{s}\right]=\frac{1}{k} \int_{r_{s}}^{\infty} k_{1}\left(r_{p}\right) H\left(r_{p}, x, t\right) d r_{p} ;
$$

thus, the inaccessible fractional flow function is

$$
f_{n s}\left[H, r_{s}\right]=1-f_{a}\left[H, r_{s}\right]=\frac{1}{k} \int_{0}^{r_{s}} k_{1}\left(r_{p}\right) H\left(r_{p}, x, t\right) d r_{p} .
$$

The system of governing equations for suspension transport in porous media consists of the mass balance equation for particles and the kinetic equations for particle retention and pore plugging.

First, the conservation law for suspended and retained particles results in the advective-capture equation for suspended and retained concentrations in the following form:

$$
\begin{array}{r}
\frac{\partial}{\partial t}\left\{\phi_{a}\left[H, r_{s}\right] C\left(r_{s}, x, t\right)+\Sigma\left(r_{s}, x, t\right)\right\} \\
+U \frac{\partial}{\partial x}\left\{C\left(r_{s}, x, t\right) f_{a}\left[H, r_{s}\right]\right\}=0 .
\end{array}
$$

Second, particle capture kinetics is obtained from the assumption that particle accumulation in the pores with given sizes is proportional to the particle flux through these pores:

$$
\begin{aligned}
\frac{\partial \Sigma\left(r_{s}, x, t\right)}{\partial t}= & \frac{U C\left(r_{s}, x, t\right) f_{a}\left[H, r_{s}\right]}{l k[H]} \\
& \times \int_{0}^{r_{s}} k_{1}\left(r_{p}\right) H\left(r_{p}, x, t\right) d r_{p} .
\end{aligned}
$$

The proportionality coefficient is equal to the capture probability per unit length of particle trajectory. The length parameter $l$ is the distance between mixing chambers in the PTMC model [32, 41].

Third, under the assumption of complete plugging, one retained particle plugs one pore and vice versa. It allows deriving the following kinetic equation for pore concentration decrease due to particle size exclusion:

$$
\begin{aligned}
\frac{\partial H\left(r_{p}, x, t\right)}{\partial t}= & -\frac{k_{1}\left(r_{p}\right)}{k} U H\left(r_{p}, x, t\right) \\
& \times \int_{0}^{r_{s}} C\left(r_{s}, x, t\right) f_{a}\left[H, r_{s}\right] d r_{s} .
\end{aligned}
$$

The system of three governing equations (9)-(11) determines the suspended and retained particle concentration distributions along with the pore concentration distribution, $C, \Sigma$, and $H$. This completes the stochastic model for suspension transport in porous media with distributed pore and particle sizes.

In the next section, the microscale system of governing equations for suspension transport in porous media will be applied to the flow of monodispersed suspension, in which case the upscale equation system is derived and the analytical solution is obtained in macroscale.

\section{Upscaling for Transport of Monodispersed Suspension}

In the case of monodispersed suspension transport in porous media with arbitrary pore size distribution, the particle concentration distribution function can be expressed using the Dirac delta function $\delta: C\left(r_{s}, x, t\right)=c(x, t) \delta\left(r_{s}-r_{s 0}\right)$, where $r_{s 0}$ is the particle size. Substitution of $C\left(r_{s}, x, t\right)$ into system (9)-(11) and integration in terms of $r_{s}$ in (9) and (10) and $r_{p}$ in (11) result in the following governing equations in macroscale:

$$
\begin{gathered}
\frac{\partial}{\partial t}\left\{\phi_{a}[H] c(x, t)+\sigma(x, t)\right\}+U \frac{\partial}{\partial x}\left\{c(x, t) f_{a}[H]\right\}=0 \\
\frac{\partial \sigma(x, t)}{\partial t}=\frac{1}{l} f_{a} f_{n s}[H] c(x, t) U \\
\frac{\partial h(x, t)}{\partial t}=-U c(x, t) f_{a} f_{n s}[H] \equiv-l \frac{\partial \sigma(x, t)}{\partial t}
\end{gathered}
$$

Equation (14) indicates the conservation of the sum of volumetric concentrations of vacant pores and retained particles, $h / l+\sigma=h_{0} / l+\sigma_{0}$, which is exactly the mathematical form for the condition of complete pore plugging.

An implicit analytical solution for pore concentration distribution function $H\left(r_{p}, x, t\right)$ is derived with the uniform initial condition for $H$ [32]: $t=0: H\left(r_{p}, x, t\right)=H_{0}\left(r_{p}\right)$. In this case, the solution $H\left(r_{p}, x, t\right)$ is equivalent to the solution 
of an ordinary-differential-integral equation $H\left(r_{p}, y\right)$

$$
H\left(r_{p}, y\right)=H_{0}\left(r_{p}\right) e^{-k_{1} y} ; \quad y=0: H=H_{0}\left(r_{p}\right),
$$

where the auxiliary function $y(h)$ is implicitly determined from the following problem as an inverse function [32]:

$$
h(y)=\int_{0}^{\infty} H_{0}\left(r_{p}\right) e^{-k_{1} y} d r_{p} ; \quad y=0: h=h_{0} .
$$

The final form of governing equations for the transport of monosize particle suspension is obtained from the system (12)-(14) and the solution for pore concentration distribution function (15)-(16). Introduction of the dimensionless parameters $X=x / L, T=U t / L \phi_{0}, C=c / c^{0}, S=\sigma / c^{0} \phi_{0}, \Lambda=$ $L f_{a} f_{n s} / l$, and $s=\phi_{a} / \phi_{0}$ leads to the following dimensionless form of governing equations:

$$
\begin{gathered}
\frac{\partial}{\partial T}\{s(S) C+S\}+\frac{\partial}{\partial X}\left\{C f_{a}(S)\right\}=0, \\
\frac{\partial S}{\partial T}=\Lambda(S) C .
\end{gathered}
$$

The initial and boundary conditions for constant injection of suspension into clean porous media are $T=0: C=S=0$; $X=0: C=1$.

The system of (17)-(18), a type of the first-order hyperbolic system [42], contains functions of saturation, accessible fractional flow, and filtration, all of which depend on retained particle concentration in porous media. The analytical solution to the system (17)-(18) subject to the initial and boundary conditions will be presented in the next section.

\section{Analytical Solution for Long-Term Monodispersed Suspension Transport}

Derivation of the analytical solution starts from expressing the suspension concentration $C$ from kinetic equation (18) in the following form:

$$
C=\frac{1}{\Lambda(S)} \frac{\partial S}{\partial T} .
$$

An auxiliary function $F(S)$ is introduced such that $C=$ $\partial F(S) / \partial T$. Substitution into (19) leads to

$$
F(S)=\int_{0}^{S} \frac{1}{\Lambda(u)} d u
$$

Mass balance equation (17) is rewritten as

$$
\frac{\partial}{\partial T}\left(s(S) \frac{\partial F(S)}{\partial T}+S\right)+\frac{\partial}{\partial X}\left(\frac{\partial F(S)}{\partial T} f_{a}(S)\right)=0 .
$$

Rearranging the second term on the left side of (21)

$$
\begin{aligned}
\frac{\partial}{\partial X}\left(\frac{\partial F(S)}{\partial T} f_{a}(S)\right) & =\frac{\partial}{\partial X}\left(\frac{\partial F(S)}{\partial S} \frac{\partial S}{\partial T} f_{a}(S)\right) \\
& =\frac{\partial}{\partial X} \frac{\partial}{\partial T}\left(\int_{0}^{S} \frac{\partial F}{\partial S}(u) f_{a}(u) d u\right) \\
& =\frac{\partial}{\partial T}\left(\frac{\partial F(S)}{\partial X} f_{a}(S)\right)
\end{aligned}
$$

and integrating (21) in terms of $T$ result in

$$
s(S) \frac{\partial F(S)}{\partial T}+S+\frac{\partial F(S)}{\partial X} f_{a}(S)=g(X),
$$

where the function $g(X)=0$ can be determined from the initial condition.

The first-order hyperbolic equation (23) has the characteristic lines satisfying the following equation:

$$
\frac{d T}{d X}=\frac{s(S)}{f_{a}(S)} .
$$

The first-order PDE (23) is degenerated to the first-order ODE along the characteristic lines (24)

$$
\frac{d F(S)}{d X}=-\frac{S}{f_{a}(S)} .
$$

From the boundary condition $C(0, T)=1=\partial F(S) / \partial T$, we have $X=0: F(S)=T$. Therefore, $F\left(S\left(0, T_{0}\right)\right)=T_{0}$ at arbitrary moment, $T_{0}$. This provides the solution for the initial retained particle concentration $S\left(0, T_{0}\right)$ at the moment $T_{0}$

$$
\int_{0}^{S\left(0, T_{0}\right)} \frac{1}{\Lambda(S)} d S=T_{0}
$$

Subsequently, we obtain the retained particle concentration profile $S(X, T)$ by integration of the ODE (25) as follows:

$$
\int_{S(X, T)}^{S\left(0, T_{0}\right)} \frac{f_{a}(S)}{S \Lambda(S)} d S=X
$$

Finally, the suspended particle concentration $C(X, T)$ is calculated from (19) by using the results of retained concentration profile $S(X, T)$ from $(27)$

$$
C(X, T)=\frac{1}{\Lambda(S)} \frac{\partial S(X, T)}{\partial T} .
$$

So far, the exact solution for long-term transport of monodispersed suspension in porous media has been derived. Application of the proposed model to a synthetic medium will be demonstrated in the next section. 


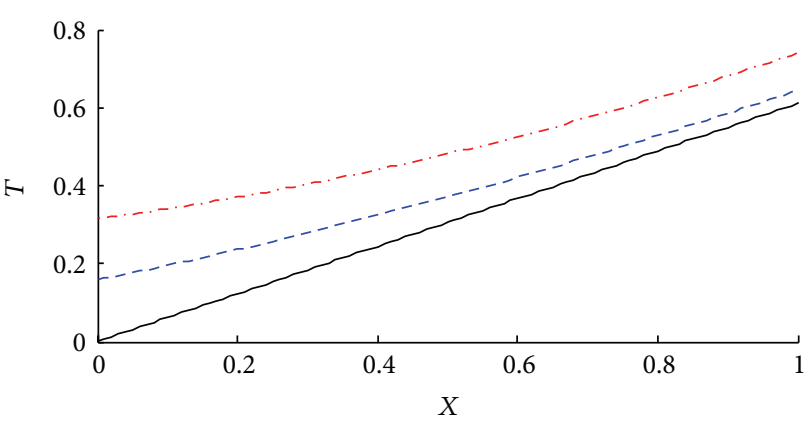

(a)

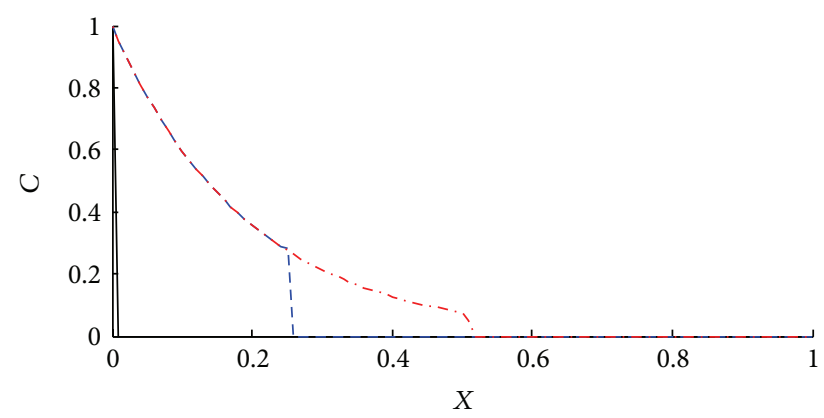

(b)

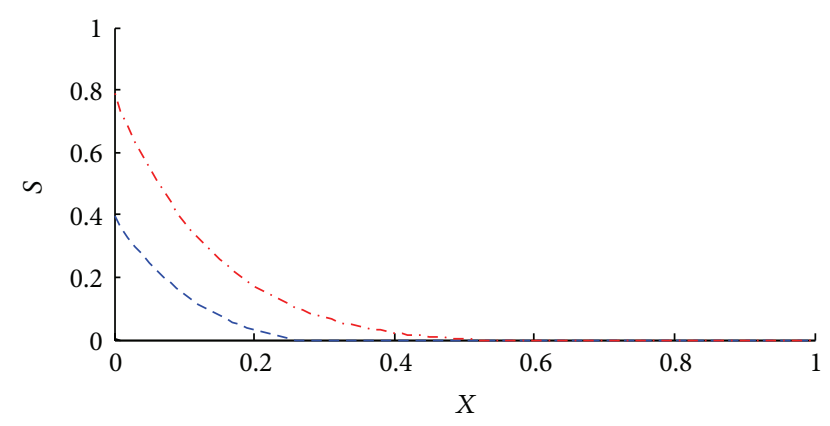

(c)

Figure 2: The exact solution for 1D deep bed filtration long-term injection problem: (a) characteristic lines and the concentration front in $(X, T)$-plane; (b) shock front in the suspended concentration profile; (c) continuous retention concentration profile.

\section{Results and Discussions}

In this section, the analytical solution derived in Section 4 is applied to a synthetic example of porous medium. The solution behaviour is analysed in detail.

Let us start from the structure of the flow pattern, shown in Figure 2. At the beginning of the suspension injection (black curves in Figure 2), the suspended particle concentration $C$ drops from unity to zero at $X=0$ and the retained particle concentration $S$ is zero everywhere, according to the initial and boundary conditions. Both the saturation $s$ and the fractional flow function $f_{a}$ are constant; therefore, the characteristic curve is straight line at $T=0$ based on (24). When the time $T>0$, the concentration front starts propagating from inlet $X=0$ along the core. In

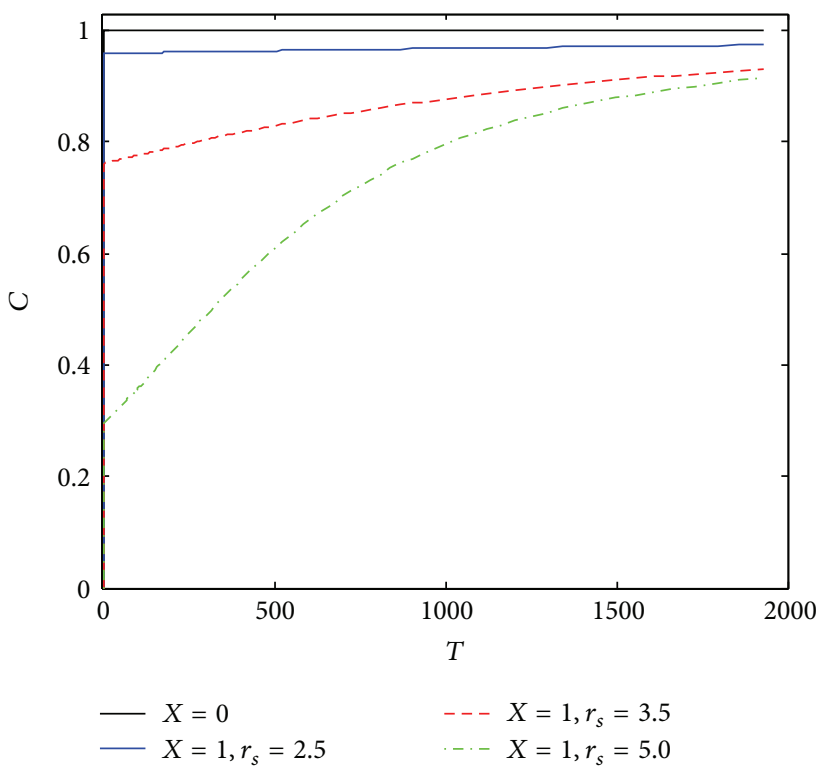

(a)

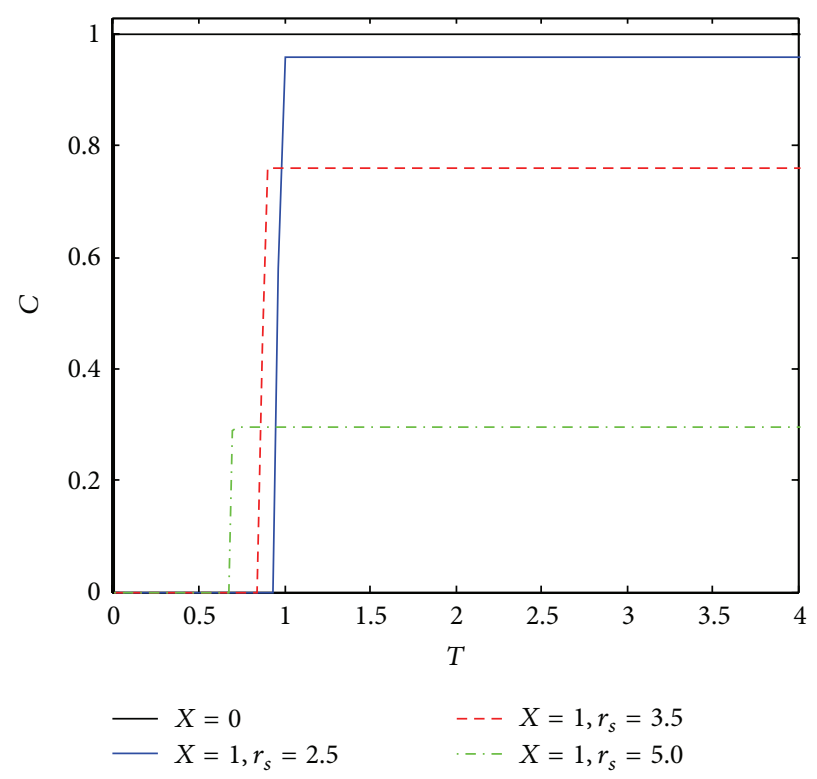

(b)

Figure 3: Suspended particle concentration at the core inlet and outlet: (a) full time scale history; (b) zoom-in at the early stage.

Figure 2, the blue dashed curves present an early stage and the red dash-dot curves correspond to a later moment. It is shown that particle concentrations $C$ and $S$ decrease along the distance $X$ behind the concentration front while remain zero ahead of the front. The characteristic curve deviates from straight line when $T>0$. The larger the time, the higher the nonlinearity.

Consider the porous medium with a log-normal pore size distribution, with the mean pore radius $4 \mu \mathrm{m}$ and the 


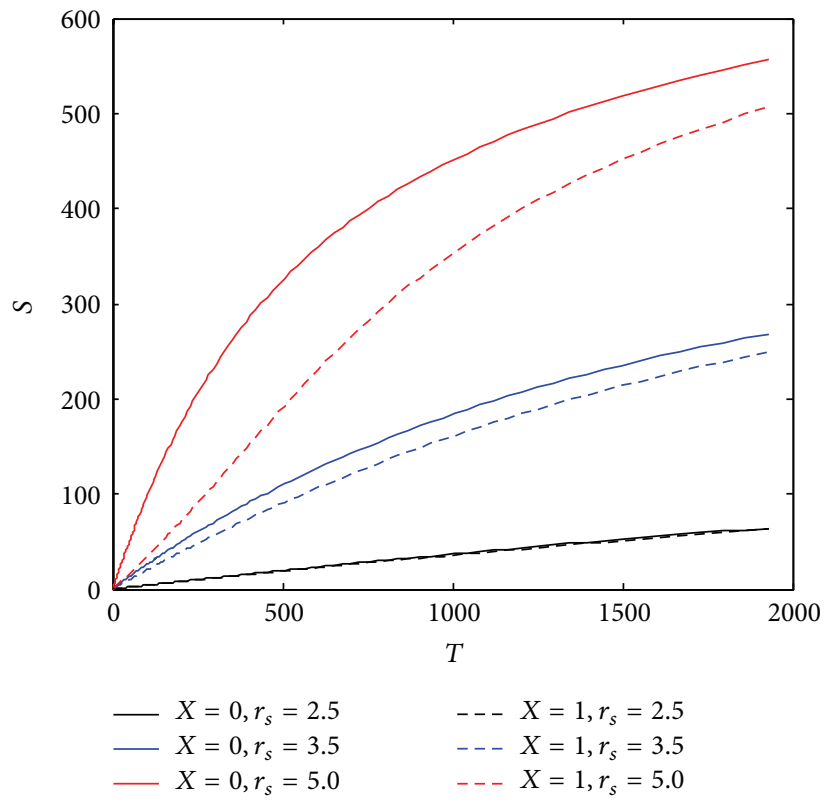

(a)

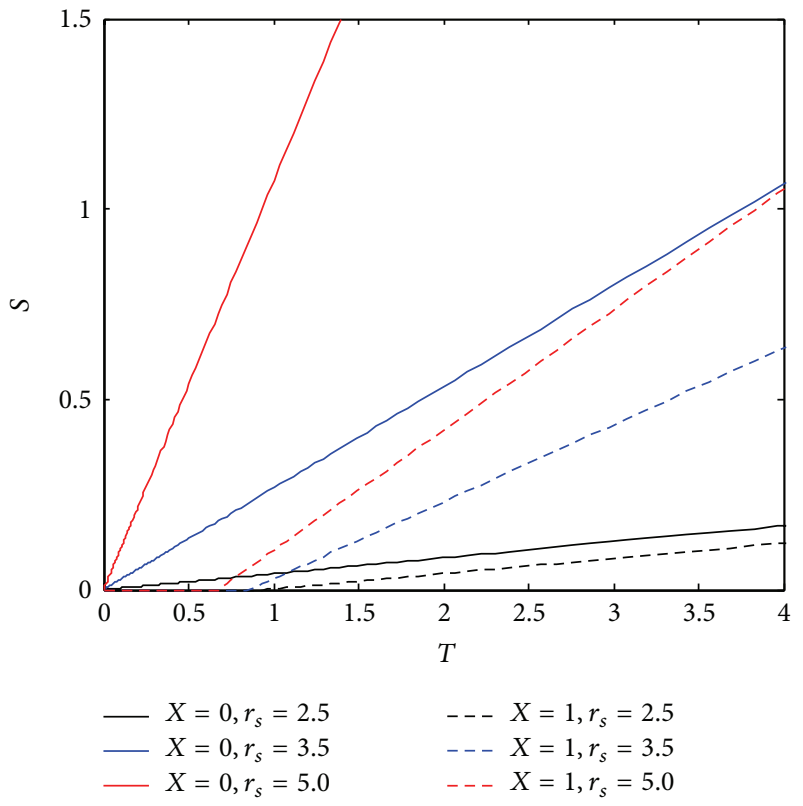

(b)

FIGURE 4: Captured particle concentration at the inlet and outlet: (a) full time scale history; (b) zoom in at the early stage.

standard deviation $2 \mu \mathrm{m}$. Three monodispersed suspensions are injected into the medium separately, with particle sizes $2.5,3.5$, and $5.0 \mu \mathrm{m}$, respectively. Suspended particle concentration profiles $C(X, T)$ are calculated from the analytical solution derived in Section 4. Figure 3(a) shows the inlet concentration $C(0, T)$ and outlet concentrations for different particle sizes $C(1, T)$ in full scale. Figure $3(\mathrm{~b})$ focuses on the early stage around the breakthrough moment. It is found that particle breakthrough occurs at $T<1$ pvi; the increase of particle size results in earlier breakthrough but lower breakthrough concentration. After breakthrough, there is a plateau of outlet concentration at small time (Figure 3(b)); then $C(1, T)$ increases gradually and approaches unity asymptotically at large time (Figure 3(a)).

The retained particle concentration profile $S$ is presented in Figure 4. The profiles $S(0, T)$ at the inlet and $S(1, T)$ at the outlet for three particle sizes are illustrated in Figure 4(a); while the zoom in at early stage is in Figure 4(b) for comparison. Particle retention at the inlet starts from $T=0$, increasing linearly with time at the early stage (Figure 4(b)). Compared to the deposition profile $S(0, T)$ at the inlet, the profile $S(1, T)$ at the outlet exhibits a delay (Figure 4(b)) due to the time required for particle travelling from inlet to outlet. The larger the particle size, the smaller the delay, indicating the earlier breakthrough for large particles, which agrees with the results shown in Figure 3. In the long time span (Figure 4(a)), the retained particle concentration increases nonlinearly with time and asymptotically approaches the maximum, which is equal to the overall concentration of pores smaller than the particle.
Figure 5 compares the evolution of the pore concentration distribution for different particle sizes. At $T=0, H=$ $H_{0}\left(r_{p}\right)$ for all three particle sizes (black curves in Figures 5(a)-5(c)). When $T>0, H\left(r_{p}, X, T\right)$ decreases with time for smaller pores $\left(r_{p}<r_{s}\right)$ due to particle size exclusion; while $H$ keeps intact for $r_{p}>r_{s}$, which means larger pores are always accessible to the particles. The pores with intermediate sizes between the minimum pore size and the particle size vanish faster with time than the smaller pores. It is because the accessible suspension flux via intermediate pores is higher than that via small pores, which leads to the larger capture rate in intermediate pores. Finally, all the pores smaller than the particle size will disappear when the time tends to infinity, which corresponds to the full plugging of small pores by the particles.

In Figure 6, the nonlinear relationship between the filtration coefficient and the retained particle concentration is presented for different particle sizes. For the same value of retained particle concentration, the larger particle size leads to the larger filtration coefficient. With the particle size fixed, the higher retention concentration causes the filtration function to deviate from the linearity, where the low retention assumption is no longer valid [41].

Fractional flow function curve obtained from PTMC model is given in Figure 7. The increase of particle size results in the decrease of both accessible porosity and accessible flow fraction. The convex shape of the curve implies that the ratio of $f_{a} / s$ increases with particle size, which corresponds to the rise of propagation speed of concentration front. It is in agreement with the results presented in Figures 3 and 4. 

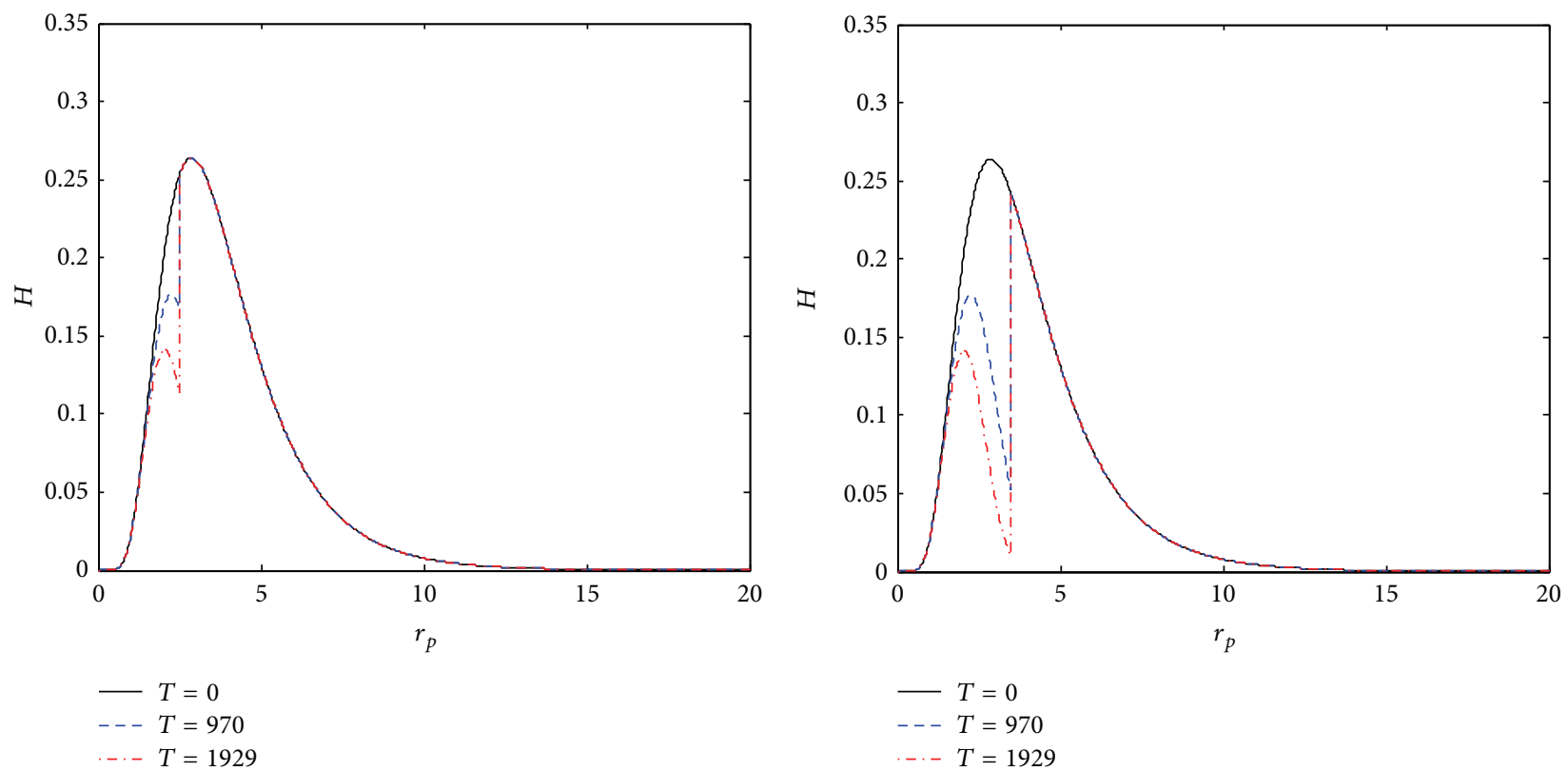

(a)

(b)

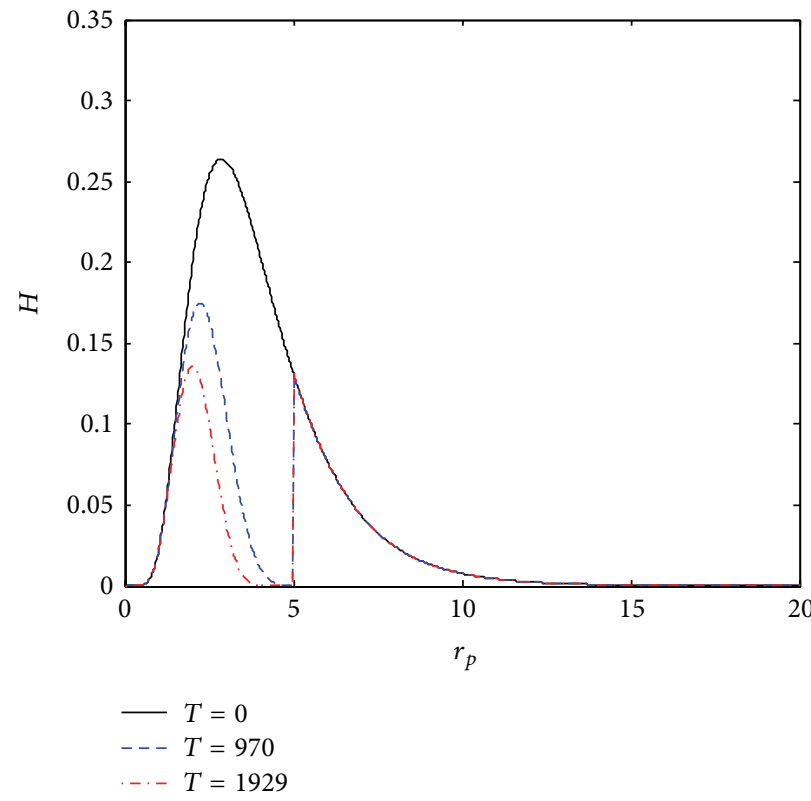

(c)

FIgURE 5: Evolution of the pore size distribution for different sizes of injected particles: (a) $r_{s}=2.5 \mu \mathrm{m}$; (b) $r_{s}=3.5 \mu \mathrm{m}$; (c) $r_{s}=5.0 \mu \mathrm{m}$.

The geometrical model of parallel capillaries intercalated by mixing chambers adopted in the current work, results in connective set of pores for any arbitrary particle size $r_{s}>0$. However, this is not the case for the real geometry of porous space. The pores smaller than the threshold value do not form an infinite cluster. The threshold effect is described by the percolation model of porous media [43-45]. Percolation description of porous space with corresponding calculations of accessibility and flux reduction factors will significantly change the form of fractional flow curve [32] and affect the solution.
The majority of deep bed filtration processes in the nature and in industry do not long as far as asymptotic stabilisation without capture. Nevertheless, these processes last longer than short-term periods of linear deep bed filtration with constant filtration coefficient [41]. The linear deep bed filtration corresponds to the case of invariant pore size distribution. This is an asymptotic case where the concentration of retained particles is negligibly smaller than the number of initial vacant pores. More exactly, the linear solution is the zero term in asymptotic expansion. The expansion could correspond to small injected concentration, 


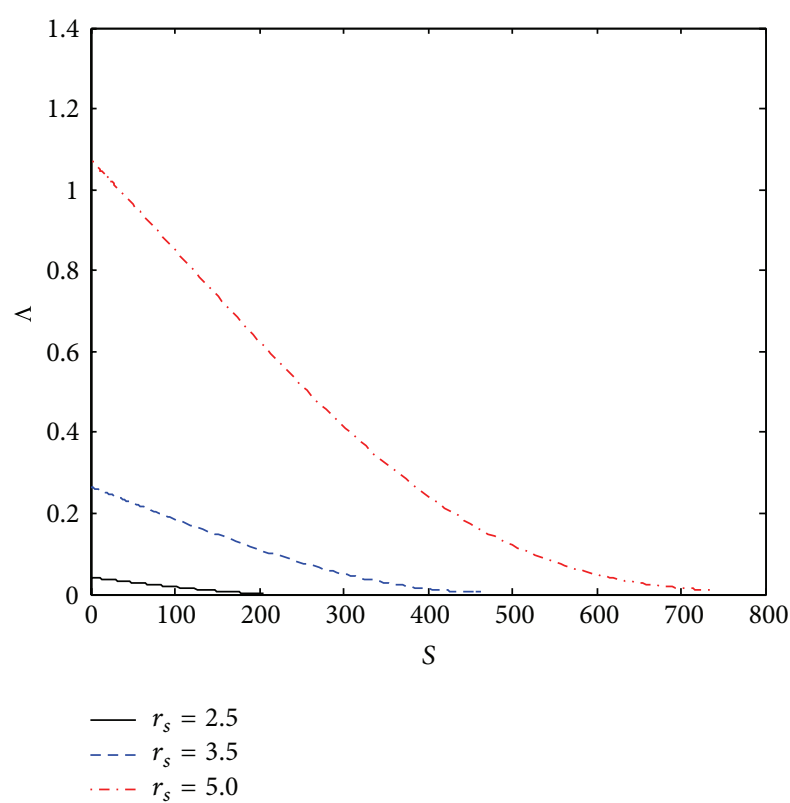

FIGURE 6: Filtration coefficient as function of captured particle concentration.

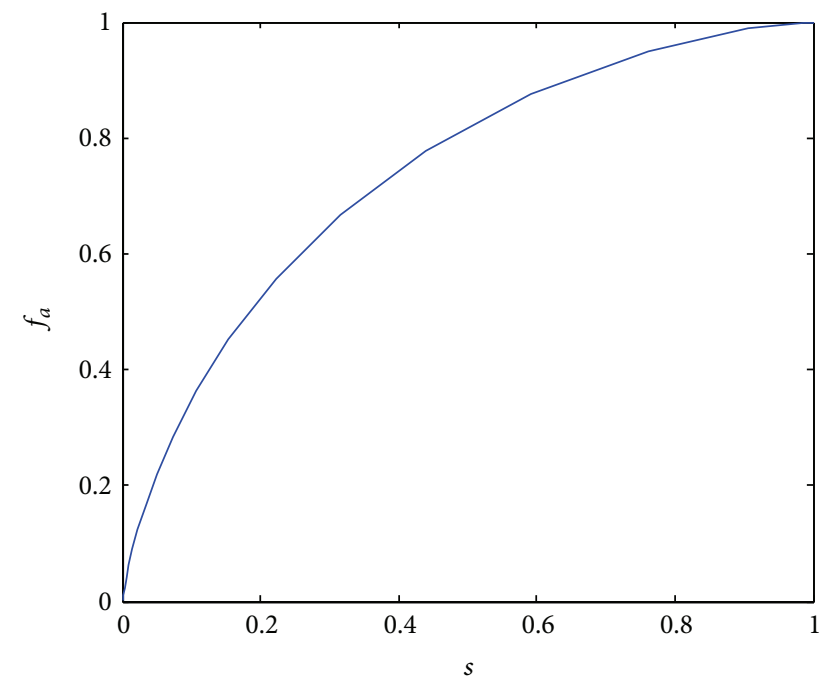

FIgURE 7: Fractional flow function for accessible flow.

or small filtration coefficient, or small times. Asymptotic solution including first term of the expansion would provide more detailed description of the intermediate stage of deep bed filtration. It is expected that the effect of full plugging of intermediate size pores (Figure 5) would be captured by the first term of asymptotic expansion.

\section{Conclusions}

Derivation of the exact solution for long-term deep bed filtration with accessibility and flux reduction allows drawing the following conclusions.

The exact solution of the upscaled problem allows for downscaling, exhibiting the dynamics for pore size distributions during the continuous particle straining.
The exact solution exhibits preferential plugging of pores with sizes equal to or below the injected particle size. The pores with radius equal to the injected particle radius disappear first. Then the smaller pores start to disappear in the sequence of their sizes. The radius interval of disappearing sizes increases with time. The lower bound of the interval turns to zero asymptotically when time tends to infinity, which corresponds to complete plugging of pores smaller than the particle size.

The larger the injected particles, the faster the breakthrough and the lower the breakthrough concentration. However, the breakthrough concentrations of all size particles tend to one with time tending to infinity, which corresponds to complete plugging of smaller pores and capture-free transport via the larger pores.

The above are the consequences of the geometric porous space model with parallel tubes, which also results in the convex fractional flow function.

\section{Acknowledgments}

The authors thank Professor Alexander Shapiro (Technical University of Denmark) for long-term cooperation on stochastic modelling of deep bed filtration. Especial thanks are due to Drs. Alex Badalyan and Themis Carageorgos (ASP) for the firm support and encouragement. The privilege of scientific contacts with Professor Y. Osipov (Moscow Civil Engineering University) and discussions of asymptotical nonlinear analysis are gratefully acknowledged.

\section{References}

[1] G. I. Barenblatt, V. M. Entov, and V. M. Ryzhik, Theory of Fluid Flows Through Natural Rocks, Kluwer Academic, Dordrecht, The Netherlands, 1990.

[2] P. Bedrikovetsky, Mathematical Theory of Oil and Gas Recovery: With Applications to Ex-USSR Oil and Gas Fields, Kluwer Academic, Dordrecht, The Netherlands, 1993.

[3] K. C. Khilar and H. S. Fogler, Migrations of Fines in Porous Media, Kluwer Academic, Dordrecht, The Netherlands, 1998.

[4] F. Civan, Reservoir Formation Damage: Fundamentals, Modeling, Assessment, and Mitigation, Gulf Professional, Amsterdam, The Netherlands, 2nd edition, 2007.

[5] J. M. Schembre and A. R. Kovscek, "Mechanism of formation damage at elevated temperature," Journal of Energy Resources Technology, Transactions of the ASME, vol. 127, no. 3, pp. 171180, 2005.

[6] F. Civan, "Non-isothermal permeability impairment by fines migration and deposition in porous media including dispersive transport," Transport in Porous Media, vol. 85, no. 1, pp. 233-258, 2010.

[7] Z. You, A. Badalyan, and P. Bedrikovetsky, "Size-exclusion colloidal transport in porous media-stochastic modeling and experimental study," SPE Journal, vol. 18, pp. 620-633, 2013.

[8] D. C. Mays and J. R. Hunt, "Hydrodynamic aspects of particle clogging in porous media," Environmental Science and Technology, vol. 39, no. 2, pp. 577-584, 2005.

[9] D. C. Mays and J. R. Hunt, "Hydrodynamic and chemical factors in clogging by montmorillonite in porous media," Environmental Science and Technology, vol. 41, no. 16, pp. 56665671, 2007. 
[10] C. Tien and B. V. Ramarao, Granular Filtration of Aerosols and Hydrosols, Elsevier, Amsterdam, The Netherlands, 2nd edition, 2007.

[11] P. Baveye, P. Vandevivere, B. L. Hoyle, P. C. DeLeo, and D. Sanchez De Lozada, "Environmental impact and mechanisms of the biological clogging of saturated soils and aquifer materials," Critical Reviews in Environmental Science and Technology, vol. 28, no. 2, pp. 123-191, 1998.

[12] M. Vidali, "Bioremediation. An overview," Pure and Applied Chemistry, vol. 73, no. 7, pp. 1163-1172, 2001.

[13] A. I. Packman and J. S. MacKay, "Interplay of stream-subsurface exchange, clay particle deposition, and streambed evolution," Water Resources Research, vol. 39, no. 4, pp. ESG41-ESG49, 2003.

[14] K.-M. Yao, M. T. Habibian, and C. R. O’Melia, "Water and waste water filtration: concepts and applications," Environmental Science and Technology, vol. 5, no. 11, pp. 1105-1112, 1971.

[15] M. Elimelech, X. Jia, J. Gregory, and R. Williams, Particle Deposition and Aggregation: Measurement, Modelling and Simulation, Butterworth-Heinemann, Oxford, UK, 1995.

[16] W. J. Weiss, E. J. Bouwer, R. Aboytes et al., "Riverbank filtration for control of microorganisms: results from field monitoring," Water Research, vol. 39, no. 10, pp. 1990-2001, 2005.

[17] N. Tufenkji, D. R. Dixon, R. Considine, and C. J. Drummond, "Multi-scale Cryptosporidium/sand interactions in water treatment," Water Research, vol. 40, no. 18, pp. 3315-3331, 2006.

[18] J. Y. Shin, R. F. Spinette, and C. R. O’Melia, "Stoichiometry of coagulation revisited," Environmental Science and Technology, vol. 42, no. 7, pp. 2582-2589, 2008.

[19] S. Torkzaban, J. Wan, T. K. Tokunaga, and S. A. Bradford, "Impacts of bridging complexation on the transport of surfacemodified nanoparticles in saturated sand," Journal of Contaminant Hydrology, vol. 136-137, pp. 86-95, 2012.

[20] S. A. Bradford, S. Torkzaban, and A. Shapiro, "A theoretical analysis of colloid attachment and straining in chemically heterogeneous porous media," Langmuir, vol. 29, pp. 69446952, 2013.

[21] J. P. Herzig, D. M. Leclerc, and P. Legoff, "Flow of suspensions through porous media-application to deep filtration," Industrial and Engineering Chemistry, vol. 62, no. 5, pp. 8-35, 1970.

[22] L. M. McDowell-Boyer, J. R. Hunt, and N. Sitar, "Particle transport through porous media," Water Resources Research, vol. 22, no. 13, pp. 1901-1921, 1986.

[23] J. N. Ryan and M. Elimelech, "Colloid mobilization and transport in groundwater," Colloids and Surfaces A, vol. 107, pp. 1-56, 1996.

[24] J. F. Schijven and S. M. Hassanizadeh, "Removal of viruses by soil passage: overview of modeling, processes, and parameters," Critical Reviews in Environmental Science and Technology, vol. 30, no. 1, pp. 49-127, 2000.

[25] T. K. Sen and K. C. Khilar, "Review on subsurface colloids and colloid-associated contaminant transport in saturated porous media," Advances in Colloid and Interface Science, vol. 119, no. 2-3, pp. 71-96, 2006.

[26] S. A. Bradford and S. Torkzaban, "Colloid transport and retention in unsaturated porous media: a review of interface-, collector-, and pore-scale processes and models," Vadose Zone Journal, vol. 7, no. 2, pp. 667-681, 2008.

[27] J. S. Chang, S. Vigneswaran, J. K. Kandasamy, and L. J. Tsai, "Effect of pore size and particle size distribution on granular bed filtration and microfiltration," Separation Science and Technology, vol. 43, no. 7, pp. 1771-1784, 2008.
[28] D. Lin, X. Tian, F. Wu, and B. Xing, "Fate and transport of engineered nanomaterials in the environment," Journal of Environmental Quality, vol. 39, no. 6, pp. 1896-1908, 2010.

[29] T. K. Sen, "Processes in pathogenic biocolloidal contaminants transport in saturated and unsaturated porous media: a review," Water, Air, and Soil Pollution, vol. 216, no. 1-4, pp. 239-256, 2011.

[30] A. C. Payatakes, R. Rajagopalan, and C. Tien, "Application of porous media models to the study of deep bed filtration," The Canadian Journal of Chemical Engineering, vol. 52, pp. 722-731, 1974.

[31] F. Kuhnen, K. Barmettler, S. Bhattacharjee, M. Elimelech, and R. Kretzschmar, "Transport of iron oxide colloids in packed quartz sand media: monolayer and multilayer deposition," Journal of Colloid and Interface Science, vol. 231, no. 1, pp. 32-41, 2000.

[32] P. Bedrikovetsky, "Upscaling of stochastic micro model for suspension transport in porous media," Transport in Porous Media, vol. 75, no. 3, pp. 335-369, 2008.

[33] A. A. Shapiro and P. G. Bedrikovetsky, "Elliptic random-walk equation for suspension and tracer transport in porous media," Physica A, vol. 387, no. 24, pp. 5963-5978, 2008.

[34] A. A. Shapiro and P. G. Bedrikovetsky, "A stochastic theory for deep bed filtration accounting for dispersion and size distributions," Physica A, vol. 389, no. 13, pp. 2473-2494, 2010.

[35] M. M. Sharma and Y. C. Yortsos, "Transport of particulate suspensions in porous media: model formulation," AIChE Journal, vol. 33, no. 10, pp. 1636-1643, 1987.

[36] M. M. Sharma and Y. C. Yortsos, "Network model for deep bed filtration processes," AIChE Journal, vol. 33, no. 10, pp. 1644$1653,1987$.

[37] M. M. Sharma and Y. C. Yortsos, "Fines migration in porous media," AIChE Journal, vol. 33, no. 10, pp. 1654-1662, 1987.

[38] A. Santos and P. Bedrikovetsky, "A stochastic model for particulate suspension flow in porous media," Transport in Porous Media, vol. 62, no. 1, pp. 23-53, 2006.

[39] A. A. Shapiro, P. G. Bedrikovetsky, A. Santos, and O. O. Medvedev, "A stochastic model for filtration of particulate suspensions with incomplete pore plugging," Transport in Porous Media, vol. 67, no. 1, pp. 135-164, 2007.

[40] L. D. Landau and E. M. Lifshitz, Fluid Mechanics, Pergamon Press, Oxford, UK, 2nd edition, 1987.

[41] P. Chalk, N. Gooding, S. Hutten, Z. You, and P. Bedrikovetsky, "Pore size distribution from challenge coreflood testing by colloidal flow," Chemical Engineering Research and Design, vol. 90, no. 1, pp. 63-77, 2012.

[42] A. P. Pires and P. G. Bedrikovetsky, "First-order hyperbolic systems of quasilinear equations: systems of conservation laws of gas dynamic type," in Handbook of NonlInear Partial Differential Equations, A. D. Polyanin and V. F. Zaitzev, Eds., pp. 1607-1620, Chapman \& Hall, Boca Raton, Fla, USA, 2nd edition, 2012.

[43] V. I. Selyakov and V. V. Kadet, Percolation Models for Transport in Porous Media with Applications to Reservoir Engineering, Kluwer Academic, Dordrecht, The Netherlands, 1996.

[44] H. Yuan, A. Shapiro, Z. You, and A. Badalyan, "Estimating filtration coefficients for straining from percolation and random walk theories," Chemical Engineering Journal, vol. 210, pp. 6373, 2012.

[45] H. Yuan, Z. You, A. Shapiro, and P. Bedrikovetsky, "Improved population balance model for straining-dominant deep bed filtration using network calculations," Chemical Engineering Journal, vol. 226, pp. 227-237, 2013. 


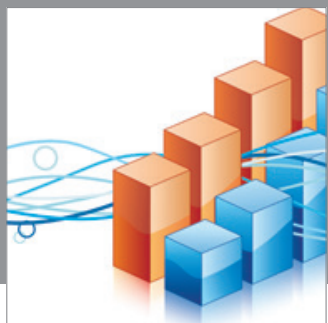

Advances in

Operations Research

mansans

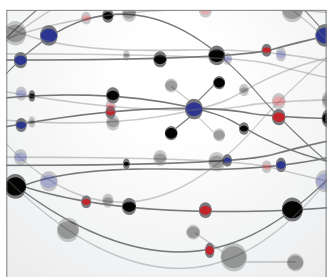

The Scientific World Journal
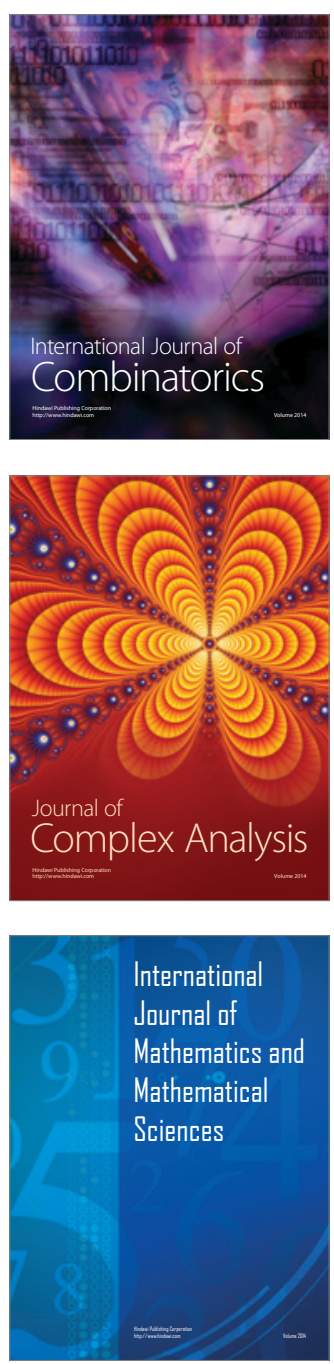
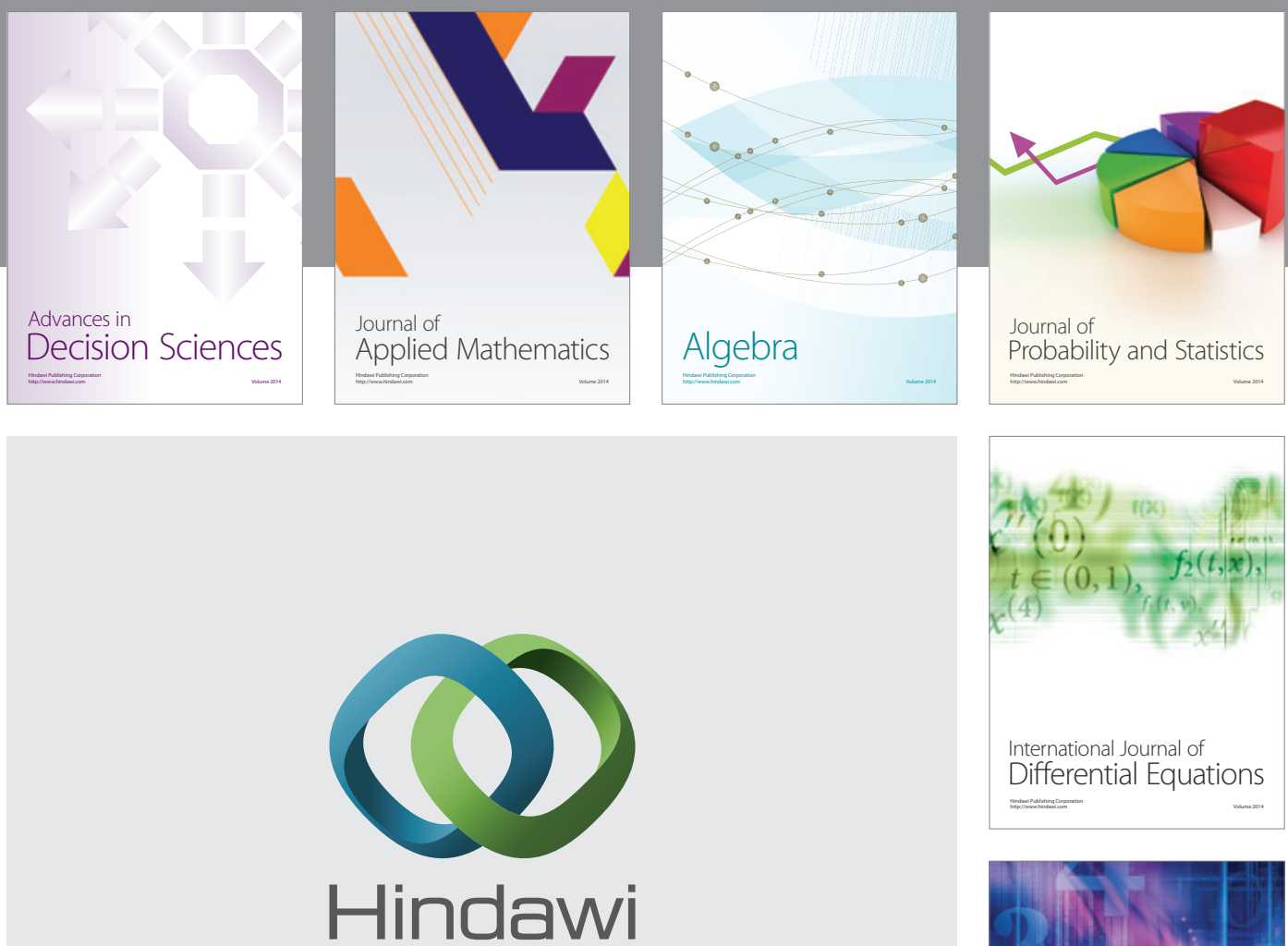

Submit your manuscripts at http://www.hindawi.com
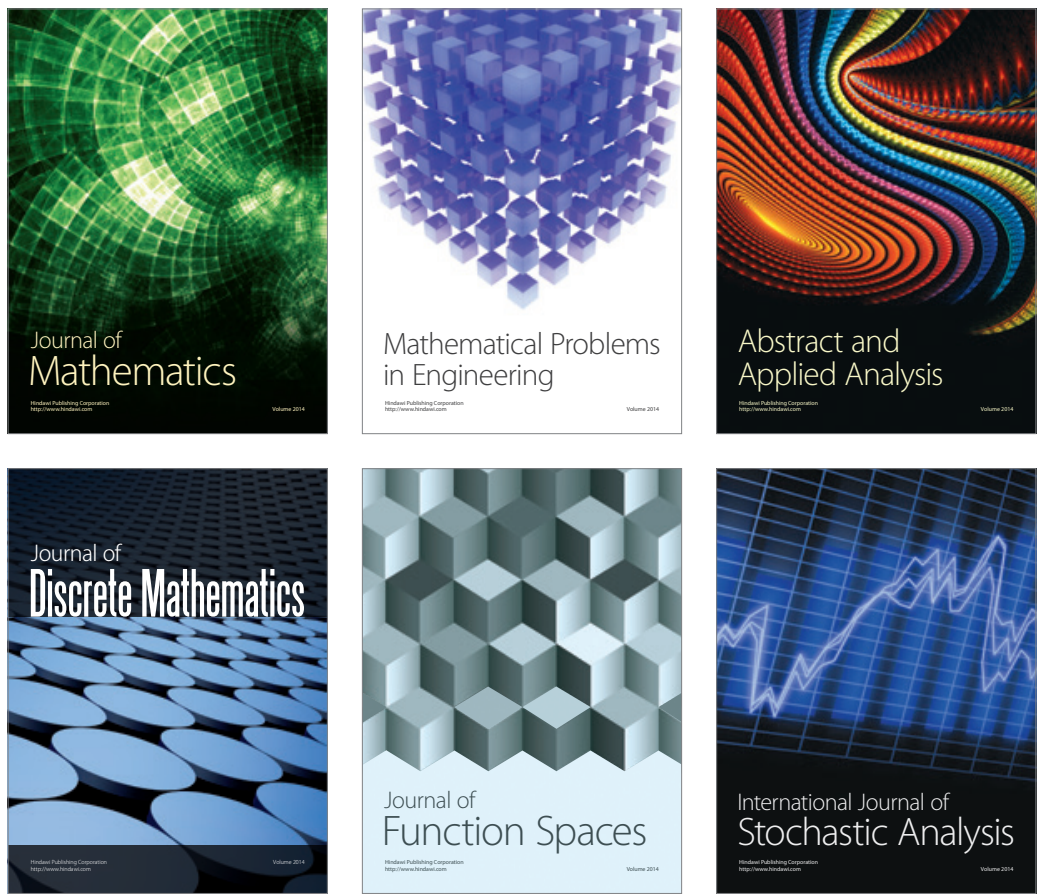

Journal of

Function Spaces

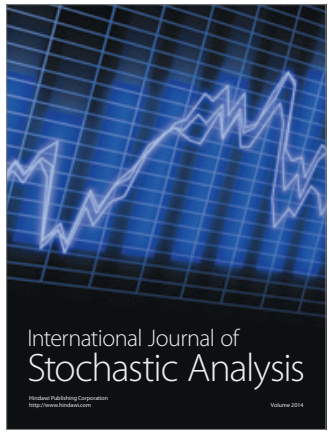

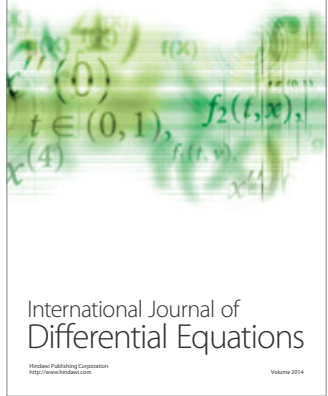
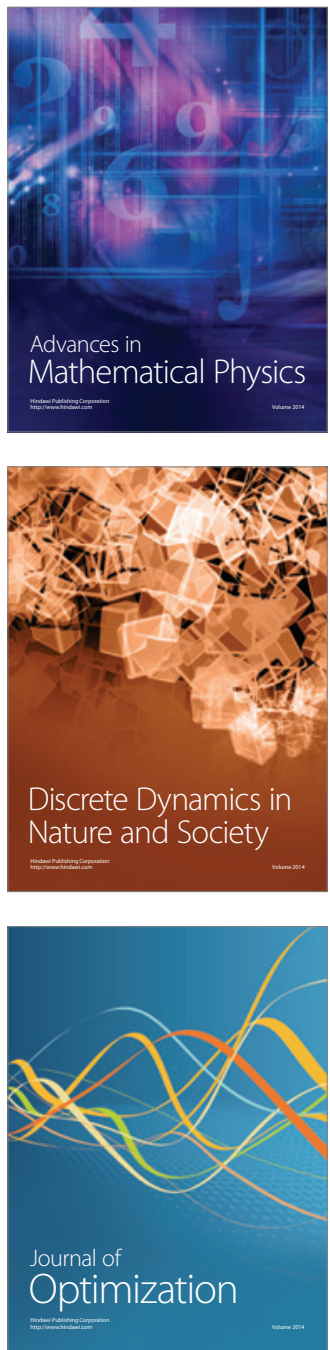